\title{
Electric Character of Strange Stars
}

\author{
R.X. Xu, G.J. Qiao \\ National Astronomical Observatories, BAC and Astronomy Department, \\ Peking University, Beijing 100781, China *† \\ Published in Chin.Phys.Lett., Vol.16, p.778
}

\begin{abstract}
Using the Thomas-Fermi model, we investigated the electric characteristics of a static non-magnetized strange star without crust in this paper. The exact solutions of electron number density and electric field above the quark surface are obtained. These results are useful if we are concerned about physical processes near the quark matter surfaces of strange stars.
\end{abstract}

PACS: 97.60.Gb, 97.60.Jd, 97.60.Sm

If strange quark matter in bulk is absolutely stable, there might be strange star [1] consisting almost completely of strange quark matter in the universe. Frustratingly, strange stars are very similar to neutron stars in their many properties, such as mass and radius. Thus, it is suggested that pulsars might be strange stars [1-3]. However, the interesting question about the nature of pulsars (neutron stars or strange stars) has not been answered with certainty even now yet.

Strange quark matter mainly consists of up, down, and strange quarks. As strange quark is a little more massive than that of up and down quarks, there are a few electrons in the chemical equilibrium of strange quark matter in order to keep the matter neutral. Hence, electromagnetic interaction as well as strong interaction results in strange quark matter. The electromagnetic force participated in makes the structure of strange quark matter more interesting and attractive. In this paper, we are to investigate this electric peculiarity of strange stars. Previously, some numerical results $[1,4]$ have been given in literature, but no exact analytical result appears.

For a static and non-magnetized strange star, the properties of strange quark matter are determined by the thermodynamic potentials $\Omega_{i}(\mathrm{i}=\mathrm{u}, \mathrm{d}, \mathrm{s}, \mathrm{e})$ which are functions of chemical potential $\mu_{i}$ as well as the strange quark mass, $m_{s}$, and the strong interaction coupling constant $\alpha_{c}[1,5]$. We use units where

${ }^{*} \mathrm{BAC}$ is CAS-PKU joint Beijing Astrophysical Center

${ }^{\dagger}$ e-mail: RXXU@bac.pku.edu.cn $\hbar=c=1$, physical quantities can be changed to be expressed in units of c.g.s. by using $\hbar c=197.327 \mathrm{fm} \cdot \mathrm{MeV}$ and $c=2.9979 \times 10^{10} \mathrm{~cm} / \mathrm{s}$. Assuming weak interaction chemical equilibrium and overall charge neutrality, we come to

$$
\begin{aligned}
& \mu_{d}=\mu_{s}=\mu, \\
& \mu_{e}+\mu_{u}=\mu, \\
& n_{e}=\left(2 n_{u}-n_{d}-n_{s}\right) / 3, \\
& n_{i}=-\frac{\partial \Omega_{i}}{\partial \mu_{i}},
\end{aligned}
$$

and the total energy density $\rho$ reads

$$
\rho=\sum_{i=1}^{4}\left(\Omega_{i}+\mu_{i} n_{i}\right)+B,
$$

where $B$ is the bag constant, and $\Omega_{i}$ referred to the Appendix in the paper by Alcock et al.[1]. The above equations (1a-1b) have only one free independent parameter, $\mu$, and establish the relations for $\rho, \mu_{i}, n_{i}$ (i $=1,2,3,4$ for $\mathrm{u}, \mathrm{d}, \mathrm{s}$, e, respectively). There are nine equations for these nine variables; therefore equ. (1a1b) are self-contained.

The calculation results from equ.(1a-1b) are shown in Fig. 1, and 2, where the number densities of $\mathrm{u}$, d, s quarks, and the quark charge density $\rho_{q}$ (in unit of Coulomb per $\mathrm{cm}^{3}$ ) are varied as a function of total energy density $\rho$. In the computation, we choose $B=(145 \mathrm{MeV})^{4}, m_{s}=200 \mathrm{MeV}$, and the renormalization point $\rho_{R}=313 \mathrm{MeV}$, both for $\alpha_{c}=0$ and $\alpha_{c}=0.3$. As $\rho$ has a mild rise variation from the outer part to the interior of a strange star [1], the number density of $\mathrm{u}, \mathrm{d}$, and s quarks increase almost in a same degree. However, the equilibrium quark charge density $\rho_{q}$ changes significantly, as $\rho$ increases (Fig. 2), which means the number of equilibrium electrons becomes smaller as one goes to a deeper region of a strange star. For a strange star with a typical pulsar mass $1.4 \mathrm{M}_{\odot}$, the total energy $\rho$ has a very modest variation with radial distance of strange star [1], from $\sim 4 \times 10^{14} \mathrm{~g}$ $\mathrm{cm}^{-3}$ (near surface) to $\sim 7 \times 10^{14} \mathrm{~g} \mathrm{~cm}^{-3}$ (near center), therefore the quark charge density $\rho_{q}$ would be 

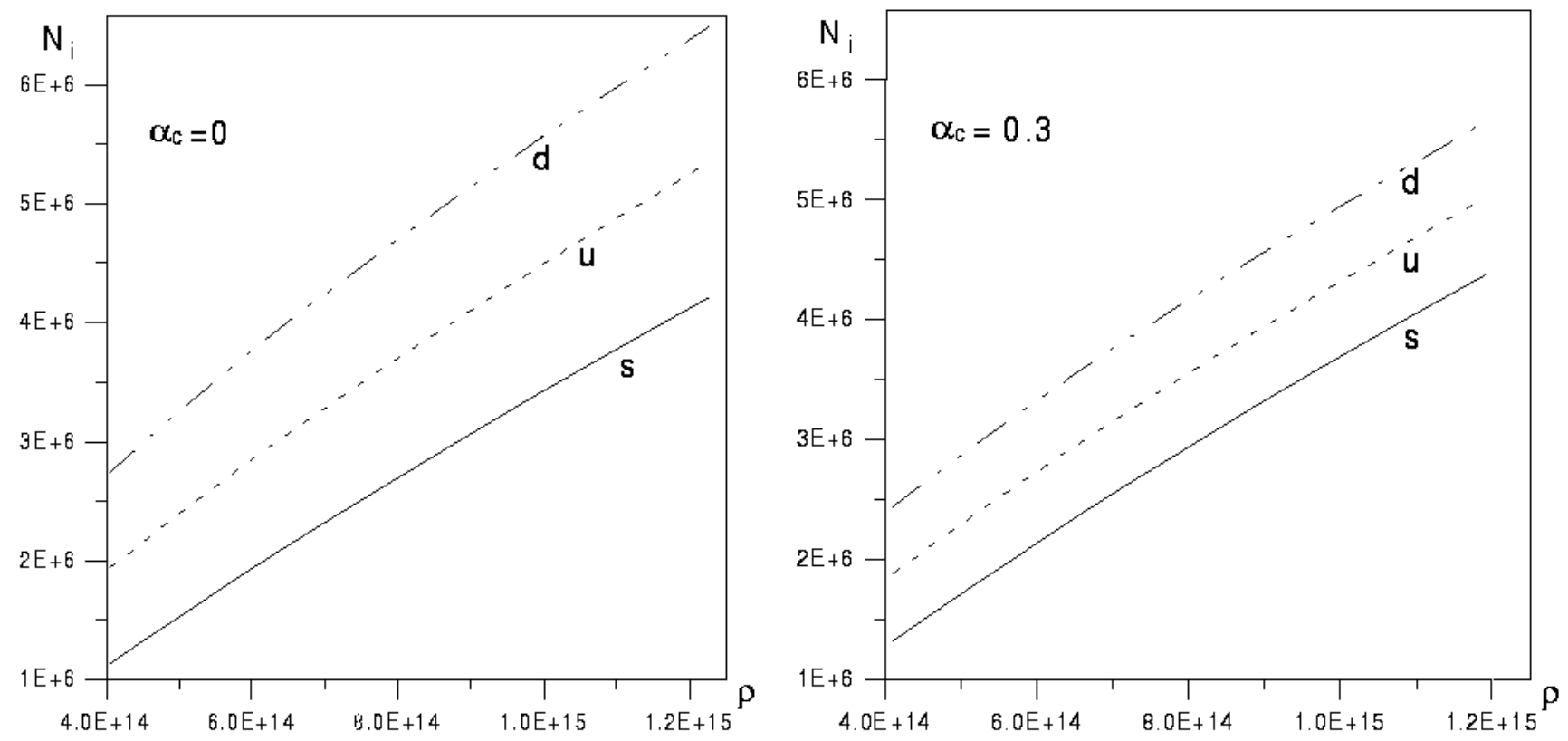

Figure 1: The number densities of $\mathrm{u}, \mathrm{d}$, and s quarks, $N_{u}, N_{d}, N_{s}$, are functions of total energy density $\rho$ (in $\left.\mathrm{g} / \mathrm{cm}^{3}\right) . N_{i}$ refers to one of $N_{u}, N_{d}$, and $N_{s}$, which are in unit of particle number per $\mathrm{cm}^{3}$. The couple constant $\alpha_{c}$ is chosen to be 0 (left) and 0.3 (right), respectively.

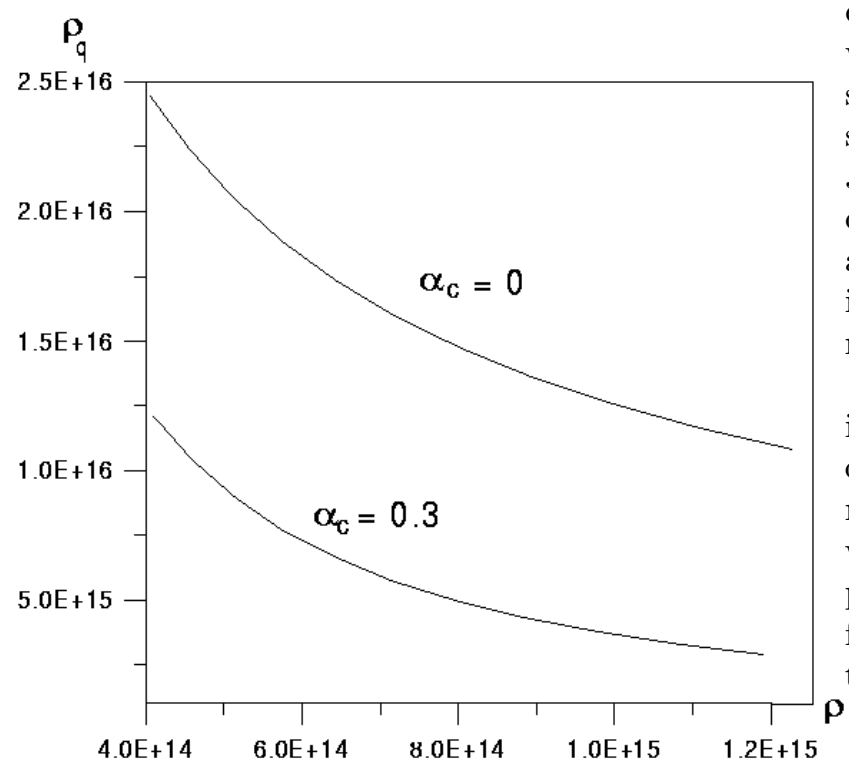

Figure 2: The quark charge density $\rho_{q}$ (in unit of Coulomb per $\mathrm{cm}^{3}$ ) decreases as a function of the total energy density $\rho$. The coupling constant $\alpha_{c}$ is chosen to be 0 and 0.3 , respectively. order of $10^{15}\left(\alpha_{c}=0.3\right)$ to $10^{16}\left(\alpha_{c}=0\right)$ Coulomb $\mathrm{cm}^{-3}$. Physically, as the Fermi energy of quarks becomes higher (for larger $\rho$ ), the effect due to $m_{s} \neq 0$ would be less important, hence, the charge density should be smaller. For a rotating magnetized strange star with typical parameters of pulsars, the GoldreichJullian space charge separated density is very small (in order of $10^{-7}$ Coulomb $/ \mathrm{cm}^{3}[6]$ ), so it is a very good approximation to neglect the space charge separation, i.e., the calculation results in Fig.1 and 2 are valid for rotating magnetized strange stars.

Since the quark matter are bounded through strong interaction (the thickness of the quark surface will be of order $1 \mathrm{fm}$ ), and the electrons are held by the quark matter electrically, hence the electron's distribution would extend beyond the quark matter surface. A simple Thomas-Fermi model has been employed to solve for this distribution [1], and the local charge distribution can be obtained by Poisson's equation

$$
\frac{d^{2} V}{d z^{2}}= \begin{cases}\frac{4 \alpha}{3 \pi}\left(V^{3}-V_{q}^{3}\right) & z \leq 0 \\ \frac{4 \alpha}{3 \pi} V^{3} & z>0\end{cases}
$$

where $\mathrm{z}$ is a measured height above the quark surface, $\alpha$ is the fine-structure constant, $V_{q}^{3} /\left(3 \pi^{2}\right)$ is the quark charge density, $V / e$ is the electrostatic potential, and the number density of electrons is given by

$$
n_{e}=\frac{V^{3}}{3 \pi^{2}} .
$$


Physically, the boundary conditions for equ.(2) are

$$
\begin{array}{ll}
z \rightarrow-\infty: & V \rightarrow V_{q}, d V / d z \rightarrow 0 \\
z \rightarrow+\infty: & V \rightarrow 0, d V / d z \rightarrow 0 .
\end{array}
$$

By a straightforward integration of equ.(2), without considering the boundary conditions, we can get

$$
\frac{d V}{d z}=\left\{\begin{array}{l}
-\sqrt{\frac{8 \alpha}{3 \pi}\left(V^{4} / 4-V_{q}^{3} V\right)+C_{1}},(z<0) \\
-\sqrt{\frac{2 \alpha}{3 \pi} V^{4}+C_{2}} \cdot(z>0)
\end{array}\right.
$$

where, $C_{1}$ and $C_{2}$ are two constants determined by the boundary conditions. Using the first condition, we get $C_{1}=\frac{2 \alpha}{\pi} V_{q}^{4}$. Using the second one, we get $C_{2}=0$. Therefore, we come to

$$
\frac{d V}{d z}=\left\{\begin{array}{l}
-\sqrt{\frac{2 \alpha}{3 \pi}} \cdot \sqrt{V^{4}-4 V_{q}^{3} V+3 V_{q}^{4}},(z<0) \\
-\sqrt{\frac{2 \alpha}{3 \pi}} \cdot V^{2} \cdot(z>0)
\end{array}\right.
$$

The continuity of equ. (4) at $z=0$ educes the result $V(z=0)=3 V_{q} / 4[1]$, and we can consider the solution of equ.(4) for $z>0$ by

$$
\frac{d V}{d z}=-\sqrt{\frac{2 \alpha}{3 \pi}} V^{2}, \text { boundary }: V(z=0)=\frac{3}{4} V_{q},
$$

hence,

$$
V=\frac{3 V_{q}}{\sqrt{\frac{6 \alpha}{\pi}} V_{q} z+4}, \quad(\text { for } z>0) .
$$

Therefore the number density of electrons is [from equ. (3)]

$$
\begin{aligned}
n_{\mathrm{e}} & =\frac{9 V_{q}^{3}}{\pi^{2}\left(\sqrt{\frac{6 \alpha}{\pi}} V_{q} z+4\right)^{3}} \\
& \sim \frac{9.49 \times 10^{35}}{\left(1.2 z_{11+4}\right)^{3}} \mathrm{~cm}^{-3},
\end{aligned}
$$

and the electric field reads

$$
\begin{aligned}
E=-\frac{d V}{d z} & =\sqrt{\frac{2 \alpha}{3 \pi}} \cdot \frac{9 V_{q}^{2}}{\left(\sqrt{\frac{6 \alpha}{\pi}} V_{q} \cdot z+4\right)^{2}} \\
& \sim \frac{7.18 \times 10^{18}}{\left(1.2 z_{11}+4\right)^{2}} \mathrm{~V} \mathrm{~cm}^{-1},
\end{aligned}
$$

where, the direction of the electric field is outward, and $V_{q}$ has been chosen to be $20 \mathrm{MeV}$ (hence $\rho_{q}=270.19$ $\mathrm{MeV}^{3}=5.63 \times 10^{15}$ Coulomb cm $\left.{ }^{-3}\right), z_{11}=z /\left(10^{-11}\right.$ $\mathrm{cm})$.

It is interesting that, from equ.(8), although the electric field near the surface is about $10^{17} \mathrm{~V} \mathrm{~cm}^{-1}$, the electric field decreases very quickly above the quark surface. The calculation of electric field shows that the electric field is $\sim 10^{11} \mathrm{~V} \mathrm{~cm}^{-1}$ when $z \sim 10^{-8}$ $\mathrm{cm}$, which means the induced electric field should be dominant when $z>10^{-8} \mathrm{~cm}$ and bare strange stars (i.e. without crust) can have magnetospheres [3]. Also the electron charge density calculated from equ.(7) decreases from $2.4 \times 10^{15}$ Coulomb cm ${ }^{-3}$ (where $\left.V=\frac{3}{4} V_{q}\right)$ at the surface to $3.3 \times 10^{-9}$ Coulomb cm ${ }^{-3}$ when $z=3 \times 10^{-3} \mathrm{~cm}$, while the Goldreich-Jullian space charge separated density is in order of $10^{-8}$ near strange stars surface.

Equ.(7) and (8) are effective in discussing the properties of strange stars near the quark surface. Two examples are given here.

Example 1: As a strong outward-directed electric field exists near the quark surface, accreted matter will be repulsed just above the surface because of Coulomb force; hence a crust around the strange quark core might be formed. The properties of this crust have sufficiently been discussed in literature $[1,4,7]$, but no simple relation to describe the crust mass and the electric gap width. Since equ. (7) can be used to study the behavior of a test charged point, by introducing a coefficient $\eta \sim 1$ denoting the effective (positive) electric charge, we can get a simple relationship of crust mass $M_{\text {crust }}$ and electric gap width $z_{\mathrm{G}}$ for typical crust and strange star values

$$
M_{\text {crust }} \sim \frac{29 \times 10^{-5}}{\left(1.2 z_{\mathrm{G}}+4\right)^{2}} \eta \quad \mathrm{M}_{\odot},
$$

where $z_{\mathrm{G}}$ is in $10^{-11} \mathrm{~cm}$. For $z_{\mathrm{G}} \ll 1$ (the length scale of strong interaction is $\sim z_{\mathrm{G}}=10^{-2}$ ), we get the maximum values of crust mass $M_{\max } \sim 1.8 \times 10^{-5} \eta \mathrm{M}_{\odot}$, which consists with previous results.

Example 2: It is said that the Ruderman-Sutherland's inner- gap model [8] has a 'user friendly' nature for explaining the observed emission properties of radio pulsars. However, the RS model has a strange virtue: it can not be applied to half of neutron stars. It is assumed in RS model that the magnetospheric charge density above the polar cap is positive, which means the rotational angle velocity $\Omega$ and the magnetic momentum $\mu$ are anti- parallel (i.e. $\boldsymbol{\Omega} \cdot \mathbf{B}<0$ ). For neutron stars at which $\boldsymbol{\Omega}$ and $\mu$ are parallel, which are called 'anti-pulsars' (i.e. $\boldsymbol{\Omega} \cdot \mathbf{B}>0$ ), the inner gap can not be formed and the inner gap model does not work. If radio pulsars are bare strange stars [3], this strange virtue does not exist again. It is argued below that inner-gap sparking can also exist for anti-pulsars if radio pulsars are strange stars without crusts.

When $\boldsymbol{\Omega} \cdot \mathbf{B}>0$, the main reason that inner-gap does not exit is that neutron star can supply infinity of charged electrons, because electrons in the neutron stars' crusts can move across the magnetic field lines. (Electrons can move across magnetic fields if their kinematic energy density $\gg$ the magnetic energy density.) But for bare strange stars, the charge electrons available to be pull out from polar-cap are limited, and the times scale to pull out all these electrons is about $10^{-5}$ s. Therefore, polar-gap sparking can also be there for 
bare strange stars. The polar-cap area $S_{\mathrm{c}}$ reads

$$
\begin{aligned}
S_{\mathrm{c}} & =\frac{2 \pi^{2} R^{3}}{c P} \\
& \sim 6.58 \times 10^{8} R_{6}^{3} \cdot P_{1}^{-1} \mathrm{~cm}^{2},
\end{aligned}
$$

where $R$ is the radius of pulsars, $R_{6}=R /\left(10^{6} \mathrm{~cm}\right), P$ is period, $P_{1}=P /(1 \mathrm{~s})$, and $c$ is the light speed. From equ. (7), the number of total electrons available to be pull out for a bare strange star is $Q_{\mathrm{ss}}$,

$$
\begin{aligned}
Q_{\mathrm{ss}} & =S_{\mathrm{c}} \cdot e \int_{Z_{14}}^{+\infty} n_{\mathrm{e}} \mathrm{d} z \\
& \sim \frac{7.25 \times 10^{14}}{\left(1.2 z_{\mathrm{c}}+4\right)^{2}} \text { Coulomb, }
\end{aligned}
$$

where $z_{\mathrm{c}}$ is a critical height in $10^{-11} \mathrm{~cm}$, and $e$ is the elementary charge. At the height of $z_{\mathrm{c}}$, the electric field from quark matter and the induced unipolar electric field are equal (i.e, electrons are not forceful there). As $z_{\mathrm{c}} \sim 6 \times 10^{3}[3], Q_{\mathrm{ss}} \sim 4.2 \times 10^{7}$ Coulomb. Therefore, the time scale to pull out all these electrons is $\tau_{\mathrm{ss}}$

$$
\begin{aligned}
\tau_{\mathrm{ss}} & =\frac{Q_{\mathrm{ss}}}{\rho_{\mathrm{GJ}} S_{\mathrm{c}} c} \\
& \sim 2.1 \times 10^{-5} \mathrm{~s},
\end{aligned}
$$

where $\rho_{\mathrm{GJ}} \sim 10^{-7}$ Coulomb $\mathrm{cm}^{-3}$ is the GoldreichJullian [6] space charge separated density at polarcap. Recently, by studying the clear features of drifting pulses from PSR B0943+10, Rankin et. al. [9] find that the regular patterns of pulses consist with RS model, but the pulsar might be an anti-pulsar which is contrary to the expectations of RS model. We argue that PSR B $0943+10$ is a bare strange star with $\boldsymbol{\Omega} \cdot \mathbf{B}>0$.

We would like to thank our pulsar group for helpful discussions.

\section{References}

[1] C. Alcock, E. Farhi, A. Olinto, Astrophys.J. 310(1986) 261

[2] O.G. Benvenuto, J.E. Horvath, H. Vucetich, Int.J.Mod.Phys. A6(1991) 4769.

[3] XU Ren-xin, QIAO Guo-jun, Chin.Phys.Lett. 15(1998) 934

[4] HUANG Yong-feng, LU Tan, Chin.Phys.Lett. 14(1997) 314

[5] K.S. Cheng, Z.G. Dai, T. Lu, Int.J.Mod.Phys. D7(1998) 139

[6] P. Goldreich, W.H. Jullian, Astrophys.J. 157(1969) 869

[7] N.K. Glendenning, C. Kettner, F. Weber, Astrophys.J. 74 (1995) 253
[8] M.A. Ruderman, P.G. Sutherland, Astrophys.J. 196 (1975) 51

[9] J. Glanz, Science, 283 (1999), 783 